\title{
SQUID Magnetometry and Magneto-Optics of Epitaxial EuSe
}

\author{
K. RumpF ${ }^{a}$, P. Granitzer ${ }^{a}$, W. Kellner ${ }^{b}$, \\ R. Kirchschlager ${ }^{c}$, S. JaneceK ${ }^{c}$, H. Pascher ${ }^{b}$ \\ AND H. KRENN ${ }^{a}$ \\ ${ }^{a}$ Institut für Experimentalphysik, Universität Graz, 8010 Graz, Austria \\ ${ }^{b}$ Experimentalphysik I, Universität Bayreuth, 95440 Bayreuth, Germany \\ ${ }^{c}$ Institut für Halbleiter- u. Festkörperphysik, Universität Linz
}

4040 Graz, Austria

\begin{abstract}
The complicated $(H, T)$-magnetic phase diagram of EuSe is caused by the critical balance between nearest and next nearest neighbour exchange interaction $\left(J_{\mathrm{NN}}=0.119 \mathrm{~K}\right.$ and $\left.J_{\mathrm{NNN}}=-0.1209 \mathrm{~K}\right)$ and leads to various spin arrangements NNSS. .., NSN..., NNS, NNN... [NS denotes opposite ferromagnetic order in adjacent (111) planes]. Beside the subtle local exchange of $5 d-t_{2 \mathrm{~g}}$ electrons and localized holes with neighbouring Eu- $4 f$ spins, obviously also the strain status influences the occurrence of these different phases. We investigate the magnetic ordering phenomenon in a strained $2.5 \mu \mathrm{m} \mathrm{EuSe}$ film on $\mathrm{BaF}_{2}$ substrate by SQUID magnetometry and magneto-optics like spectral Faraday- and Kerr-effect measurements for temperatures from $2 \mathrm{~K}$ up to $200 \mathrm{~K}$ and for magnetic fields up to $5 \mathrm{~T}$. The magneto-optical probe monitors the local environment of the photoexcited electron-hole pair, called magnetic exciton, located within a ferromagnetic surrounding (photoinduced magnetic polaron), whereas the integral magnetization measured by SQUID is most sensitive to long-range magnetic ordering. In spite of the dissimilarity of measurement techniques we find an influence of the long-range magnetic order (e.g. of the NNS- or NNN-matrix) on the non-resonant Kerr reflection. The complementarity of SQUID and magneto-optical methods is stringent only in the (resonant) spectral regime, where magnetic polarons are formed.
\end{abstract}

PACS numbers: 75.50.Pp, 78.20.Ls, 07.55.Jg

\section{Introduction}

Among the traditional magnetic semiconductors EuX ( $\mathrm{X}=\mathrm{O}, \mathrm{S}, \mathrm{Se}, \mathrm{Te})[1]$ the metamagnetic EuSe plays an exceptional role: the exchange interaction be- 
tween nearest neighbour (NN) localized $4 f^{7}$-Eu-spins (hereafter referred to as $f$-spins) is nearly cancelled by the second nearest neighbour (NNN) antiferromagnetic interaction. The corresponding $\mathbf{E u}-\mathrm{Eu}$-exchange constants are $J_{\mathrm{NN}}=0.119 \mathrm{~K}$ and $J_{\mathrm{NNN}}=-0.1209 \mathrm{~K}[2]$. As a result of this competition the conduction electron in EuSe excited in an absorption process becomes a prominent candidate for a self-trapped magnetic polaron with characteristic red-shifts in absorption and luminescence spectra [3, 4]. The equal strength of counteracting exchange effects provides also a rich magnetic phase diagram in dependence on temperature and magnetic field: in bulk crystals a (type-II) antiferromagnetic NSNS-ordering in subsequent (111)-lattice planes is reported [1] for low temperatures $(T<1.8 \mathrm{~K})$ and for low magnetic fields ( $B<0.05 \mathrm{~T}$ ), a ferrimagnetic NNSNNS-ordering for intermediate temperatures and fields $(1.8 \mathrm{~K}<T<4.6 \mathrm{~K}, 0.1 \mathrm{~T}<B<2.5 \mathrm{~T})$ and another (type-I) antiferromagnetic NNSSNNSS-ordering close to the Curie-transition temperature $T_{\mathrm{c}}=4.6 \mathrm{~K}$. Tensile mechanical strain as naturally exerted in epitaxial layers (like in our sample of $2.5 \mu \mathrm{m}$ EuSe on $\mathrm{BaF}_{2}$ substrate due to thermal strain) could substantially modify the magnetic phases. In EuTe an applied pressure of 17 GPa transforms the originally antiferromagnetic state into a ferromagnetic one [5], similar effects of strengthening of ferromagnetic order are expected in EuSe. We present in this paper a mainly experimental, but comprehensive study of magnetization experiments (SQUID magnetometry, magneto-optic Faraday-, Kerr rotation) over a wide range of temperatures $(2-200 \mathrm{~K})$ and magnetic fields $(0-5 \mathrm{~T})$, to extract the essential magnetic features of strained EuSe.

\section{SQUID magnetometry}

The magnetic state of $\mathbf{E u S e} / \mathrm{BaF}_{2}$ has been monitored by DC magnetization $(M)$ measurements using a Quantum Design MPMS-XL-7 SQUID magnetometer, mapping $M(B, T)$ in a wide magnetic field $(B)$ and temperature $(T)$ range. Figure 1a shows the in-plane magnetization from $1.8 \mathrm{~K}$ up to temperatures $\geq T_{\mathrm{c}}$, Fig. 1b the high-temperature range $20 \mathrm{~K} \leq T \leq 200 \mathrm{~K}$. For the latter case, the paramagnetic limit (labelled as Curie-Weiss fit in the figure) holds with high accuracy if the lower truncation of the $T$-fit is set not lower than $20 \mathrm{~K}$. If one guesses $T_{\mathrm{c}} \cong 5 \mathrm{~K}$ from the turn-down of curves in Fig. 1a, the fitted paramagnetic Curie-Weiss temperature $\theta_{c}=7.6 \mathrm{~K}$ exceeds considerably $T_{c}$, which indicates a violation of the commonly used mean-field description. The occurrence of different phases is evident by inspection of the $M(T)$-curves in the region II (Fig. 1a), where the curvature is altered for $B_{\mathrm{c}} \geq 0.2 \mathrm{~T}$. This is interpreted as critical field for a transition from the ferrimagnetic NNSNNS-phase to the ferromagnetic NNNNNN-phase. A simple calculation in which the external Zeeman-energy surmounts the exchange energy for the reversal of S-spin to N-spin yields: $2 g \mu_{\mathrm{B}} S B_{\mathrm{c}} \geq 12 J_{\mathrm{NN}}+6\left|J_{\mathrm{NNN}}\right|$ with $B_{\mathrm{c}} \geq 0.23 \mathrm{~T}$ in good agreement with the experiment. A separate zoom will be performed in region I (Fig. 1a) for a possible indication of a type-II antiferromagnetic phase transition in Fig. 3. 

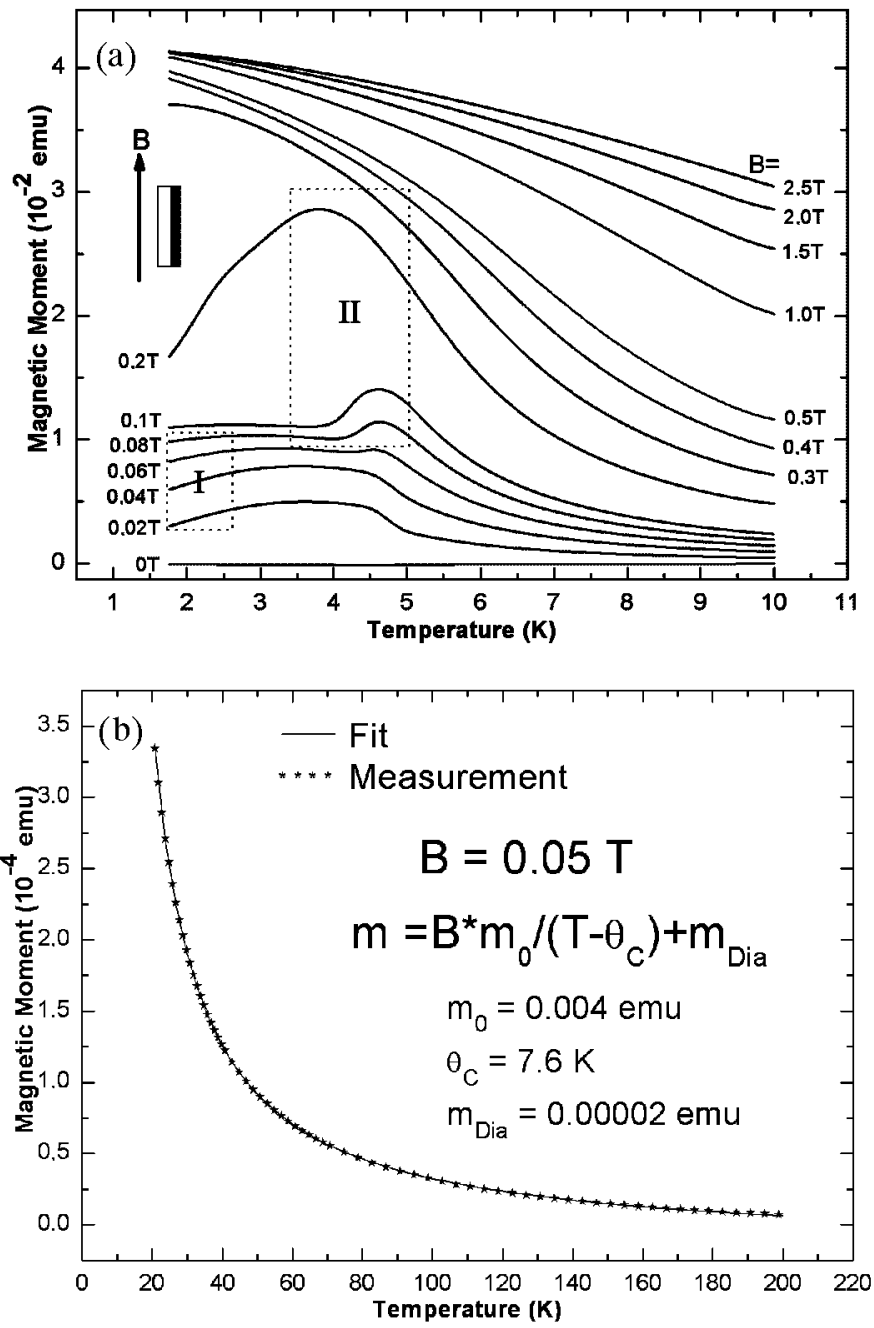

Fig. 1. Magnetization measured by SQUID for in-plane magnetic fields (a) up to $2.5 \mathrm{~T}$ and $10 \mathrm{~K}$, (b) up to $200 \mathrm{~K}$ for $B=0.05 \mathrm{~T}$ together with a Curie-Weiss fit.

An exact determination of the Curie temperature from Fig. 1a is ambiguous, since for thin samples sufficient signal is only obtained for rather high magnetic fields ( $>0.02 \mathrm{~T}$ ) with a concomitant rounding of the $M(T)$ characteristics. Therefore we have fitted Arrott plots [6] to the data in both temperatusre regions I and II, taking into account that EuSe belongs (beyond mean-field approximation) to the Heisenberg universality class. Figure 2 a shows for various temperatures (region II) the plot $M^{1 / \beta}=a(B / M)^{1 / \gamma}-b\left(T-T_{c}\right.$ ) as straight lines to $B$-data points $(B>0.1 \mathrm{~T})$. It demonstrates the validity of the Heisenberg approach in such moderate magnetic fields (otherwise saturation leads to a turnaround of the 

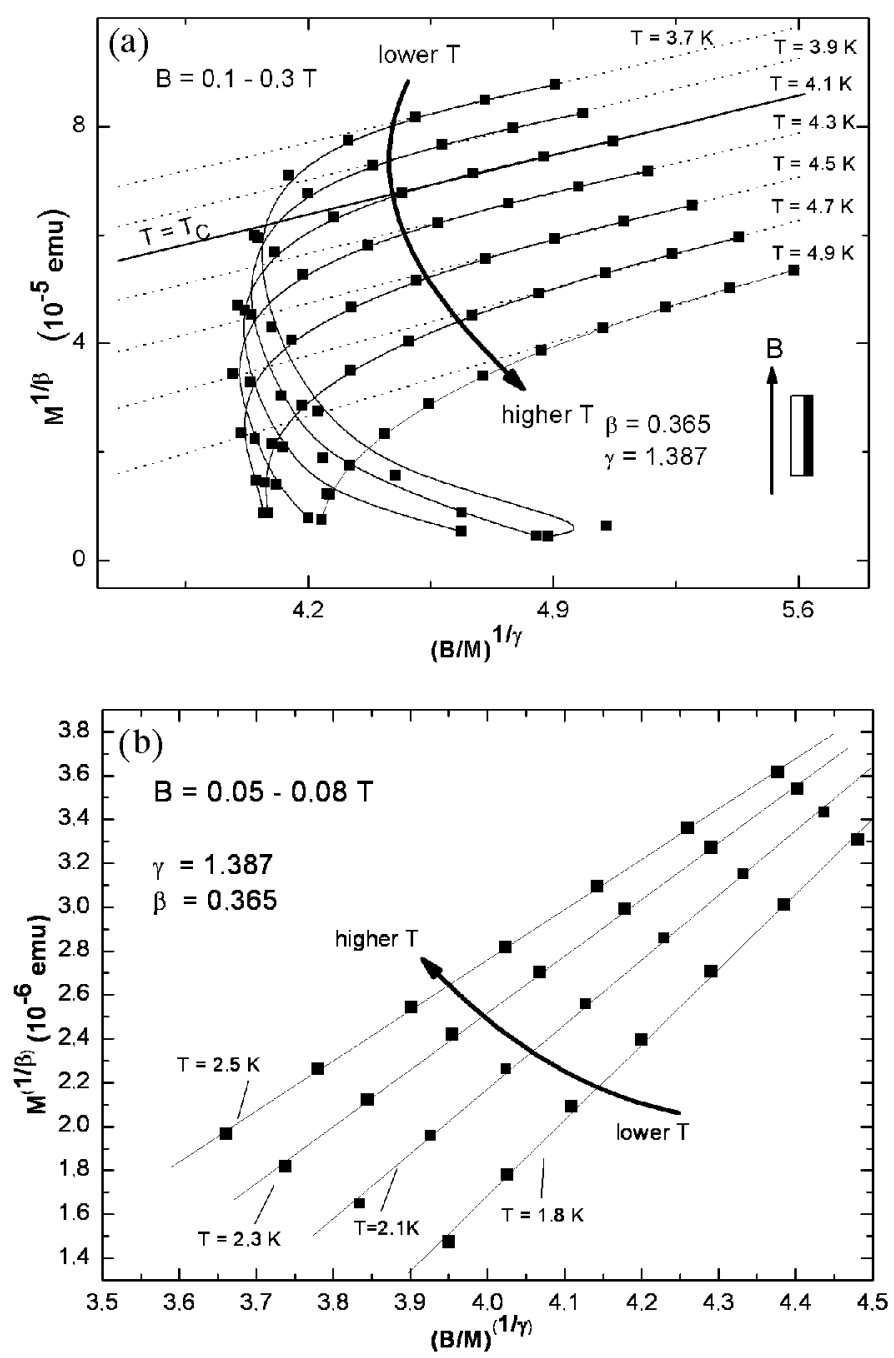

Fig. 2. Arrott plots using the Heisenberg critical exponents in the temperature range $T=3.9-4.9 \mathrm{~K}$ with linear extrapolation to determine the critical temperature $T_{\mathrm{c}}=4.1 \mathrm{~K}(\mathrm{a})$, Arrott plots in the low temperature range $T=1.8-2.5 \mathrm{~K}(\mathrm{~b})$. Let us note the reversed ordering of plots with varying temperature between (a) and (b).

Arrott plots). The line which intersects (by extrapolation) the point of origin designates the $\left(T=T_{\mathrm{c}}\right)$ isotherm. We obtain $T_{\mathrm{c}}=4.1 \mathrm{~K}$, slightly lower than bulk $T_{\mathrm{c}}=4.6 \mathrm{~K}$. In addition, all extrapolated Arrott isotherms are strictly parallel to each other which exclude biquadratic contributions to the exchange Hamiltonian as quoted in bulk EuSe [7]. Figure 2b shows Heisenberg Arrott plots in the low field/low temperature range (I), for which type-II antiferromagnetic ordering is expected. Surprisingly Arrott plots fit the data very well also in this $T$-range, 


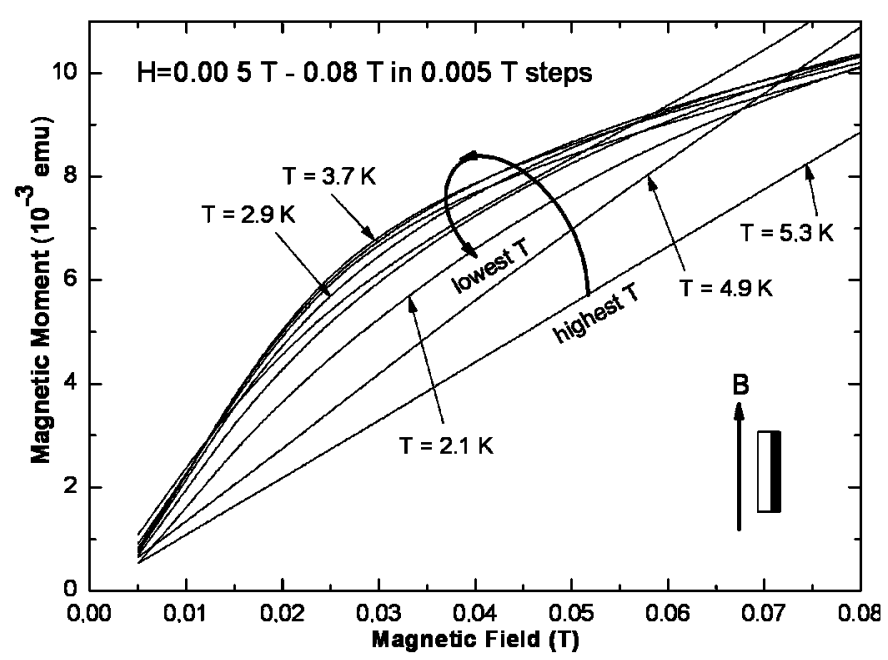

Fig. 3. Magnetization measured by SQUID as a function of magnetic field in the field regime $(B=0-0.08 \mathrm{~T}$ ) for temperatures from $5.3 \mathrm{~K}$ down to $2.1 \mathrm{~K}$. Let us note the non-monotonicity with temperature.

which advises a predominance of the ferrimagnetic phase in contrast to bulk EuSe. However, the sequence of plots from lower to higher temperature is reversed with respect to Fig. 2a and the slope changes. Both findings indicate a tendency to antiferromagnetic ordering. A definite ordering temperature cannot be revealed from these plots since they intersect the abscissa without crossing the point of origin.

The tendency toward antiferromagnetic NSNS-ordering can be directly proved by measuring the magnetization at sufficiently low magnetic fields $(B<0.08 \mathrm{~T})$, as shown in Fig. 3. For $T=5.3 \mathrm{~K}$ the linear paramagnetic response is measured, whereas for temperatures down to $3.7 \mathrm{~K}$ the magnetization increases monotonically with a tendency to saturation of the NNSNNS-phase. Below $T=3.7 \mathrm{~K}$ the slope again decreases which can only be explained by some compensation of magnetic moments with decreasing temperature due to formation of antiferromagnetic clusters. However, the experiment does not prove the existence of a long-range spontaneous antiferromagnetic ordering (at least for $T>2.1 \mathrm{~K}$ ).

The $B$-dependent magnetization for various temperatures is shown in Fig. $4 \mathrm{a}$ (with detailed zoom for low fields in Fig. 4b). In strong fields $(>0.5 \mathrm{~T})$ the magnetic order tends to ferromagnetic (NNNN) saturation, whereas for $B<0.3 \mathrm{~T}$ near $T=2.1 \mathrm{~K}$ the ferrimagnetic NNSNNS plateau is reached at exactly $1 / 3$ of the ferromagnetic saturation as expected. Due to the strain in the layer the NNSNNS structure is progressively pushed to the ferromagnetic NNNN state for increasing $T$ toward $4.5 \mathrm{~K}$. The up-turn of the $T=2.1 \mathrm{~K}$ curve near $B=0.2 \mathrm{~T}$ demonstrates the instability of the ferrimagnetic phase in good agreement with our former estimate for $B_{\mathrm{c}}=0.15 \mathrm{~T}$. 

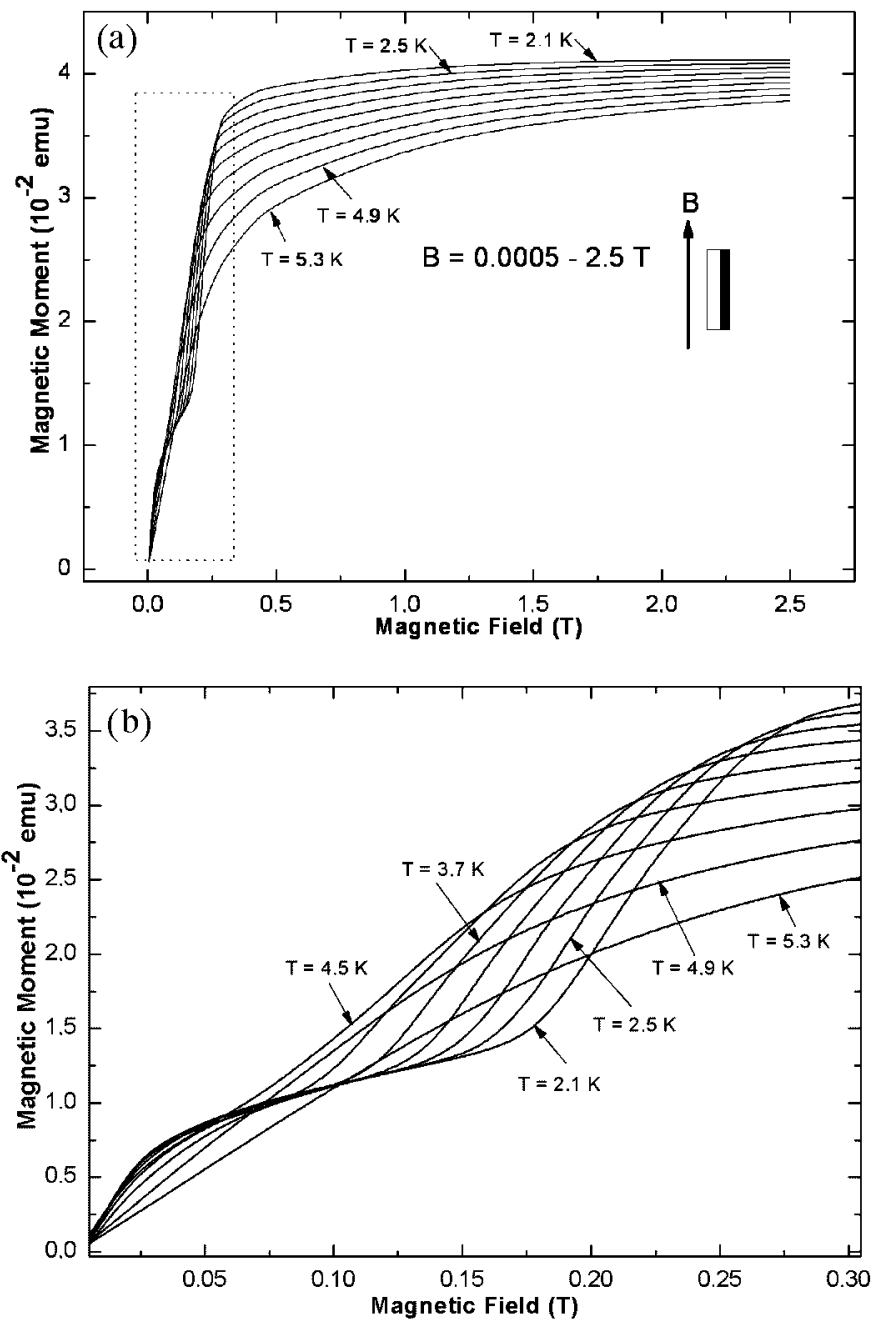

Fig. 4. (a) Full magnetization scan measured by SQUID up to $2.5 \mathrm{~T}$ for temperatures from $2.1 \mathrm{~K}$ up to $5.3 \mathrm{~K}$. The ferrimagnetic phase is shown in the dashed rectangle; (b) enlarged scale for the ferromagnetic phase, as depicted by the rectangle in panel (a).

\section{Magneto-optics}

The integral magnetization measured by SQUID suffers from possible paramagnetic impurity signals and from overwhelming diamagnetic contribution of the $\mathrm{BaF}_{2}$ substrate (mostly severe in thin film samples). Albeit such a handicap can be excluded in our experiments, magneto-optical studies represent a more local probe of magnetization, which is of particular importance in metamagnetic EuSe with a tendency to magnetic polaron formation. In the magnetic exciton model $[8,4]$ the electronic transition $4 f^{7} \rightarrow 4 f^{6} 5 d\left(t_{2 \mathrm{~g}}\right)$ lifts an electron to the more delocalized $5 d$ 
orbital. It is bound by exchange interaction to the spin-polarized hole left behind in the $4 f$ state. The adjacent Eu atoms of this hole become spin-polarized and their lattice positions are shifted (relaxed) toward the hole, forming the photoinduced localized magnetic polaron (PILMP). Interestingly, the energy of $5 d$ electron recombination with the $4 f$ hole (monitored by photoluminescence) is strongly red-shifted by the PILMP trapping. Magneto-optics in absorption does not directly deal with this strong red-shift effect near $T_{\mathrm{c}}$ and at low fields (thus the absorption energy gap is $2.1 \mathrm{eV}$ at $4.2 \mathrm{~K}$, in contrast to the emission energy gap $1.47 \mathrm{eV}$ at $2 \mathrm{~K}$ for the PILMP-photoluminescence process). Nevertheless, the Faraday rotation as shown in transmission in Fig. 5 is reminiscent to such a picture since the increasing $B$-field induces a red-shift of $0.24 \mathrm{eV}$ (as seen from the crosses in the spectra of Fig. 5), especially evident in the critical regime $T \approx T_{\mathrm{c}}$. Also a fine

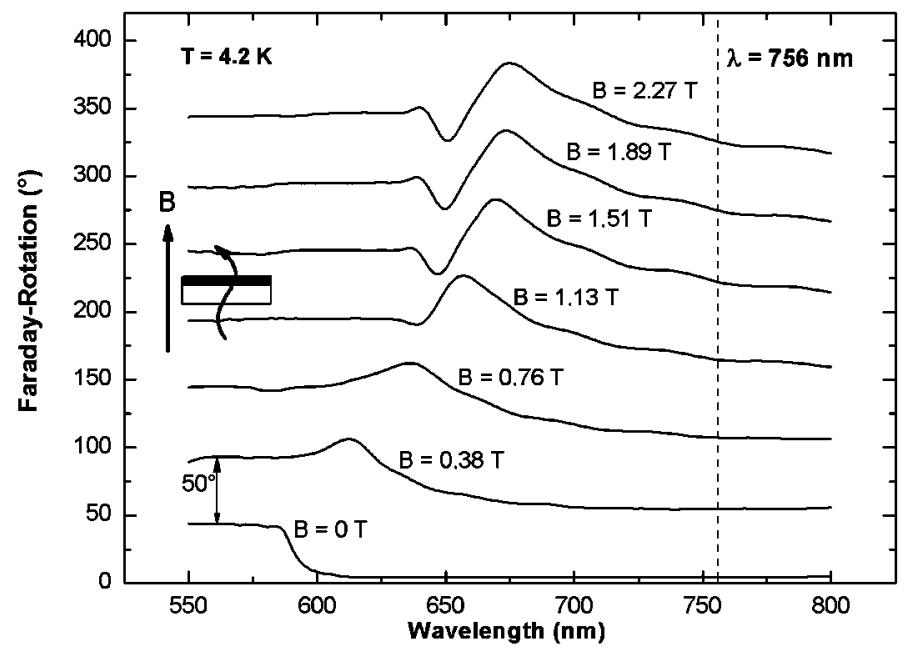

Fig. 5. Faraday rotation as a function of near-band-gap wavelength (for EuSe) at various magnetic fields. The several plots are shifted to each other by $50^{\circ}$ for better visibility. The dashed line at $\lambda=756 \mathrm{~nm}$ is a reference wavelength for the Kerr spectrum shown in Fig. 6.

structure to the left of the crosses is resolved in the topmost curves for $B>1.51 \mathrm{~T}$, which reflects a concomitant magnetic-field-induced lattice distortion, mediated by electron-phonon and spin-phonon, i.e. spin orbit (SO), interaction of the excited $5 d$ electron [3]. The dashed-dotted line denotes the spectral position where supplementing Kerr-rotation spectra have been measured as a function of magnetic field. The wavelength $\lambda=756 \mathrm{~nm}(1.64 \mathrm{eV})$ is sufficiently below the fundamental gap to avoid direct influences from PILMP- and SO-effects. Figure 6 shows magnetization curves measured by Kerr rotation at this wavelength. For a magnetic field $B \approx 0.7 \mathrm{~T}$ (marked by an arrow) a slight bump is observed for temperatures between $2 \mathrm{~K}$ and $3.5 \mathrm{~K}$, which corresponds to the ferrimagnetic plateau in 
Fig. 4b. Since no magnetic polaron is formed in this spectral region, Faraday rotation probes solely the circular birefringence induced by the long-range magnetic order as it is also measured by the SQUID. Let us note that in our magneto-optic experiments the magnetic field is perpendicular to the sample in contrast to the SQUID experiment where the field is in-plane! Taking into account the demagnetization effect, the spectrum of Fig. 6 can be rescaled to the correct built-in magnetic field $B_{\mathrm{i}}$ which equates the field applied in the SQUID experiment. An independent check has been performed (but not shown) by rotating the sample about $90^{\circ}$ in the SQUID pick-up coil.

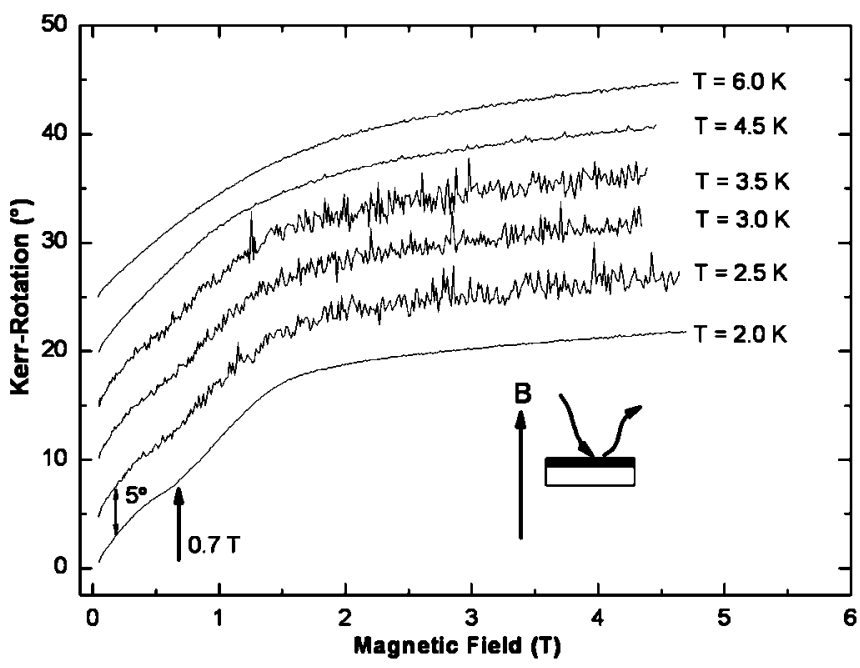

Fig. 6. Kerr rotation as a function of magnetic field for various temperatures at a wavelength $\lambda=756 \mathrm{~nm}$ in the transparent regime of EuSe. For better visibility the plots are shifted by $5^{\circ}$ each.

In summary, SQUID and magneto-optic (MO) experiments provide similar results as far as spectral dependencies in the magneto-optical transitions are carefully distinguished. Due to the local formation of the magnetic exciton, the immediate vicinity of the photocreated electron-hole pair is probed in the near-band-gap MO experiment. Resonant absorption generates a ferromagnetic particle (magnetic polaron) centred around the localized electron-hole pair, therefore the MO spectra reveal more or less ferromagnetic behaviour irrespective of ferri- or antiferromagnetic matrices, present in metamagnetic EuSe. However, the long-range magnetic order can be also resolved in a thin epitaxial EuSe film by non-resonant $M O$, as demonstrated in Fig. 6. SQUID data are always collected under the action of a magnetic field. This paper proves the Arrott plot as a useful tool for the evaluation of magnetization data in finite fields. Epitaxial EuSe is under tensile strain on $\mathrm{BaF}_{2}$ substrate, which strengthens the ferrimagnetic ordering near $T_{c}$ on the cost of the NNSS-phase. The latter is quenched by the lattice distortion and spin-orbit 
interaction. The (type-I) NNSS-antiferromagnetic phase near $T_{c}$ as observed in bulk EuSe could not be detected in the strained EuSe film, but the experiments provide some evidence for the other kind of (type-II) antiferromagnetic order at transition temperatures comparable to the bulk.

\section{Acknowledgments}

The support of the work by the Österr. Fonds zur Förderung der wiss. Forschung under grant No. P15397 is gratefully acknowledged, as well as the supply with samples by Dr. Springholz, University of Linz.

\section{References}

[1] A. Mauger, C. Godart, Phys. Rep. 141, 52 (1986).

[2] T. Janssen, Phys. Kond. Matt. 15, 142 (1972).

[3] J. Schoenes, Z. Phys. B 20, 345 (1975); J. Phys.-Cond. Matt. 15, S707 (2003).

[4] M. Umehara, J. Magn. Magn. Mater. 187, 177 (1998); Phys. Rev. B 65, 205208 (2002)

[5] I.N. Goncharenko, I. Mirabeau, Phys. Rev. Lett. 80, 1082 (1998).

[6] A. Aharoni, Introduction to the Theory of Ferromagnetism, Clarendon Press, Oxford 1996, p. 80.

[7] U. Köbler, R. Mueller, L. Smardz, D. Maier, K. Fischer, B. Olefs, W. Zinn, Z. Phys. B 100, 497 (1996).

[8] T. Kasuya, CRC Crit. Rev. Solid State Sci. 3, 189 (1972). 